\title{
Evaluación de parámetros de bienestar animal en equinos de tiro de comunidades indígenas Mapuche en la Región de la Araucanía, Chile
}

\author{
Evaluation of animal welfare parameters in draught horses of Mapuche \\ indigenous communities in the Araucanía Region, Chile \\ Christian Tuemmers ${ }^{1,2}$, Carolina Mora ${ }^{1}$, Gerardo Quezada ${ }^{1}$, \\ Angélica Saldivia ${ }^{1}$, Oscar Venegas ${ }^{1}$
}

\section{Resumen}

La creciente preocupación por un trato respetuoso hacia los animales de trabajo establece la importancia del bienestar animal en Medicina Veterinaria. La Región de la Araucanía posee una gran cantidad de sectores rurales y la tracción animal se encuentra ampliamente extendida. Con el objetivo de evaluar el estado de bienestar animal de los equinos de tiro se seleccionaron al azar 134 caballos para analizar su conformación, condición corporal, alteraciones en piel y tegumentos, patologías presentes y medidas hipométricas. El $80.5 \%$ presentó hallazgos patológicos, en donde el $69.4 \%$ estuvo relacionado con los cascos y herrajes, el $19.4 \%$ asociados a la piel, el $46 \%$ presentó condición corporal óptima y el $44 \%$ presentó una conformación física adecuada para las labores de tiro. Se estableció que los principales problemas que afectaron el bienestar animal de los equinos del estudio tienen relación con patologías podales, conformación física inadecuada y falta de aplicación de protocolos de manejo sanitario.

Palabras clave: bienestar animal; equinos de tiro; condición física; medidas hipométricas; patologías

\section{AbSTRACT}

The growing concern for a respectful treatment of working animals establishes the importance of animal welfare in Veterinary Medicine. The Region of Araucanía has several rural sectors and animal traction is widely spread. In order to evaluate the animal welfare state of the draught horses, 134 individuals were randomly selected to analyze their

\footnotetext{
${ }^{1}$ Facultad de Recursos Naturales, Departamento de Medicina Veterinaria y Salud Pública, Universidad Católica de Teтuco, Teтuco, Chile

${ }^{2}$ E-mail: ctuemmers@uct.cl
}

Recibido: 3 de septiembre de 2018

Aceptado para publicación: 26 de febrero de 2019 
conformation, body condition, alterations in skin and teguments, existing pathologies and hypometric measurements. The results showed that $80.5 \%$ presented pathological findings, where $69.4 \%$ were related to the hooves and horseshoes, $19.4 \%$ associated with the skin, $46 \%$ presented optimal body condition and $44 \%$ presented a physical conformation suitable for draught work. It was established that the main problems that affected the animal welfare of the equines of the study are related to foot pathologies, inadequate physical conformation and lack of application of sanitary management protocols.

Key words: animal welfare; draught horses; physical condition; hypometric measurements; pathologies

\section{INTRODUCCIÓN}

En los países en vías de desarrollo se encuentra la mayor cantidad de equinos, con alrededor del $60 \%$ del total de la población mundial (Pritchard et al., 2005), que según datos de FAOSTAT (2014) se acerca a 60 millones. De estos, el 55\% (32 millones) habita en el continente americano. La mayoría de estos animales se destinan principalmente a la tracción animal, contribuyendo a la agricultura familiar (Pritchard et al., 2005; Upjhon et al., 2014); sin embargo, no están exentos de una pobre condición corporal, problemas respiratorios, afecciones dentales y podopatologías (Rimbaud et al., 2006; Swann, 2006; Burn et al., 2010a), y en muchos casos no cuentan con tratamientos ni asistencia veterinaria de forma oportuna (Pritchard et $a l .$, 2005), traduciéndose en una condición deficiente de bienestar animal (BA) (Pearson y Krecek, 2006; Rimbaud et al., 2006; Tadich et al., 2008; Burn et al., 2010b).

El número de equinos en Chile se acerca a los 350000 ejemplares (FAOSTAT, 2014), teniendo como denominador común con otros países del centro y sur de América que son utilizados principalmente para labores agrícolas, transporte de personas y de carga. En la zona sur de Chile y particularmente en los sectores rurales de la Región de la Araucanía existe gran cantidad de comunidades indígenas Mapuche cuya actividad económica se basa en la agricultura fa- miliar (ODEPA, 2015), donde los equinos de tiro representan un aspecto clave en términos de sustentabilidad. Sin embargo, tal como ocurre en otros países, estos animales son mantenidos en condiciones deficientes (Pérez et al., 1993; Mac-Leod 1999; Tadich et al., 2008) y los propietarios no poseen las condiciones económicas apropiadas para costear manejos sanitarios ni tratamientos veterinarios (Mac-Leod 1999; Pearson y Krecek, 2006; Tadich et al., 2008).

La creciente preocupación por las condiciones en que se desenvuelven estos animales ha originado un renacimiento en el debate sobre el bienestar de los animales (Requejo, 2010; Hockenhull, y Whay, 2014). El concepto de bienestar de los animales se refiere a su estado físico y mental en armonía con la naturaleza o el entorno (Palmer y Sandoe, 2011; OIE, 2017). Según Broom (2011) «el bienestar de un individuo es su estado, respecto a sus intentos de enfrentar el ambiente en que se encuentra». Este concepto relaciona la fisiología, comportamiento, sentimientos y patologías que pueden presentarse como respuesta al ambiente (Korte et al., 2007), especialmente cuando se altera el comportamiento y rutina del animal, afectando aspectos como la alimentación y el descanso (Broom, 2011).

La utilización de equinos de tiro en el sur de Chile ha sido motivo de diversos estudios, especialmente en la Región de los Ríos (Pérez et al., 1993; Mac-Leod, 1999; Sáez 
et al., 2013; Lanas, 2015); sin embargo, la información sobre este tipo de caballos resulta escasa o inexistente en otras regiones del país. El objetivo del presente estudio fue evaluar el estado de bienestar animal en equinos de tiro de Comunidades Mapuche de la comuna de Padre Las Casas, región de la Araucanía, caracterizando su conformación física y clasificando las patologías presentes.

\section{Materiales y Métodos}

El estudio fue de tipo descriptivo, transversal y observacional. Se recopiló información del total de caballos de tiro de comunidades indígenas Mapuche que asistieron al policlínico de terreno en la Comuna de Padre las Casas, entre agosto y diciembre de 2016 y entre marzo y mayo de 2017. Se trabajó con 134 equinos pertenecientes a 110 propietarios. Los caballos de tiro de comunidades mapuche, si bien son mestizos, tienden a ser similares al caballo criollo chileno, aunque un poco más robustos y en la gran mayoría de los casos se asemejan a equinos de tiro liviano. Son utilizados principalmente para labores agrícolas y transporte de personas donde el tiempo de trabajo y el peso que cargan varía considerablemente entre individuos. Suelen mantenerse en pradera durante el día y estabulados durante la noche. Su alimentación base es la pradera, ya sea natural o establecida, la cual es suplementada con granos de cereales en función del poder económico del propietario.

Los datos se obtuvieron mediante la aplicación de una pauta de evaluación, que es una adaptación de la descrita por Tadich (2006) para el tipo de trabajo y animales utilizados en Chile, la cual se basa en el protocolo usado por Pritchard et al. (2005). Incluye información obtenida a partir de un examen clínico general donde se analiza la condición corporal, alteraciones en piel, tegumentos, podopatologías, medidas hipométricas, así como mediciones indirectas de bienestar a través de una encuesta a los propietarios, donde se consideran aspectos de alimentación (tipo, cantidad y frecuencia), comportamiento (estereotipias y conductas no deseadas), características de las actividades de tiro realizadas (descripción, frecuencia, duración y tipo de carruaje), manejo sanitario (protocolos de aplicación de antiparasitarios y vacunas) y manejo de herraje (frecuencia y tipo de herraje).

La edad fue determinada a partir de la información entregada por el propietario y corroborada mediante cronometría dentaria, de acuerdo con Cardona y Álvarez (2018). Para la determinación de la condición corporal se utilizó una escala de 1 a 5 (Carroll y Huntington, 1988). La determinación de las medidas hipométricas se realizó con una cinta métrica flexible de plástico graduada en centímetros. Para realizar las mediciones, los equinos debían estar libres de elementos de anclaje para labores de tiro. Se colocaron de tal manera que la masa corporal se repartiera uniformemente en las cuatro extremidades. Se tomaron las siguientes mediciones, según Pérez et al. (1993):

- Alzada: desde la altura de la cruz hasta el suelo, paralelo al miembro anterior izquierdo.

- Longitud escápulo-isquial: desde la porción craneal de la tuberosidad mayor del húmero en sentido craneocaudal hasta la tuberosidad isquiática.

- Perímetro torácico: se posicionó la cinta métrica caudal a la cruz, descendiendo por los planos costales inmediatamente posteriores a los codos, completando la circunferencia en la zona inferior del tórax.

- Perímetro metacarpiano: Se midió el perímetro del metacarpo a nivel de la diáfisis.

Los datos fueron organizados en una planilla en Microsoft ${ }^{\circledR}$ Excel. Los resultados para las distintas observaciones se analizaron mediante estadística descriptiva sobre una muestra representativa y se expresaron en base a números totales y porcentajes. 


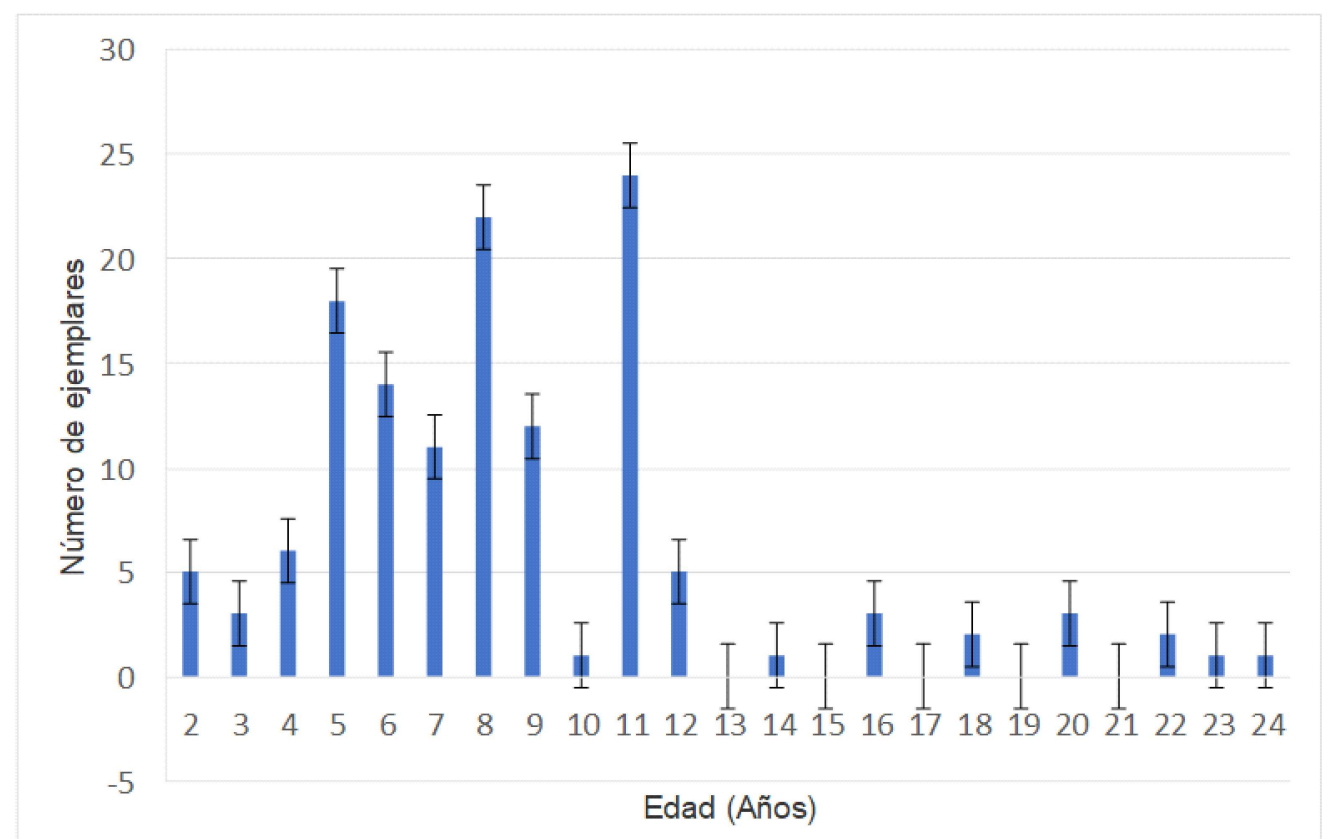

Figura 1. Distribución etaria de equinos de tiro de comunidades Mapuche en la Región de la Araucanía, Chile (2016-2017)

\section{Resultados}

El 43.2\% (58/134) fueron machos castrados, $39.5 \%$ fueron hembras $(53 / 134) \mathrm{y}$ $17.1 \%(23 / 134)$ correspondió a machos enteros. Los animales presentaron una edad promedio de 8.5 años $( \pm 4.42)$ (Figura 1$)$. La condición corporal obtuvo una puntuación de $1-3$ en el $34 \%$, de 3 en el $46 \%$ y $3-5$ en el $20 \%$ de los animales.

Se determinó la presencia de hallazgos patológicos en el 80.5\% (108/134) de los equinos, encontrando más de uno en seis ejemplares. Del total de animales afectados el $69.4 \%(75 / 108)$ presentaba alteraciones en cascos y herrajes, $19.4 \%(20 / 108)$ lesiones en piel, 9.2\% (10/108) mostraba problemas de comportamiento como estereotipias y conductas anormales, 7.4\% (8/108) claudicaciones de diverso origen y $0.9 \%(1 / 108)$ tenía problemas respiratorios (Figura 2).
El 37\% presentó un correcto herraje, mientras que el $63 \%$ restante presentó diversos tipos de alteraciones como herraje incompleto $(75 \%)$. De estos, los herrajes sobredimensionados alcanzaron un $44 \%$ y los herrajes subdimensionados un $25 \%$. Además, el $15 \%$ no presentaban herraje. Otras alteraciones encontradas fueron onicomicosis, fisuras de la muralla, encastillamiento, sobrecre-cimiento lateral o medial y laminitis (Figura 3), aunque no se pudo establecer el origen y curso de esta última. El 35\% de los equinos presentaban más de una alteración en los cascos y en un $22 \%$ hasta tres alteraciones.

El 24\% de los propietarios manifestó llevar un manejo antiparasitario por cuenta propia y de forma estricta, el $45 \%$ de los propietarios indicó que atendía a sus equinos en operativos veterinarios gratuitos que se realizan dos veces en el año. El 31\% restante no recibía antiparasitarios y en ningún ejemplar se aplicaban programas de vacunaciones. 


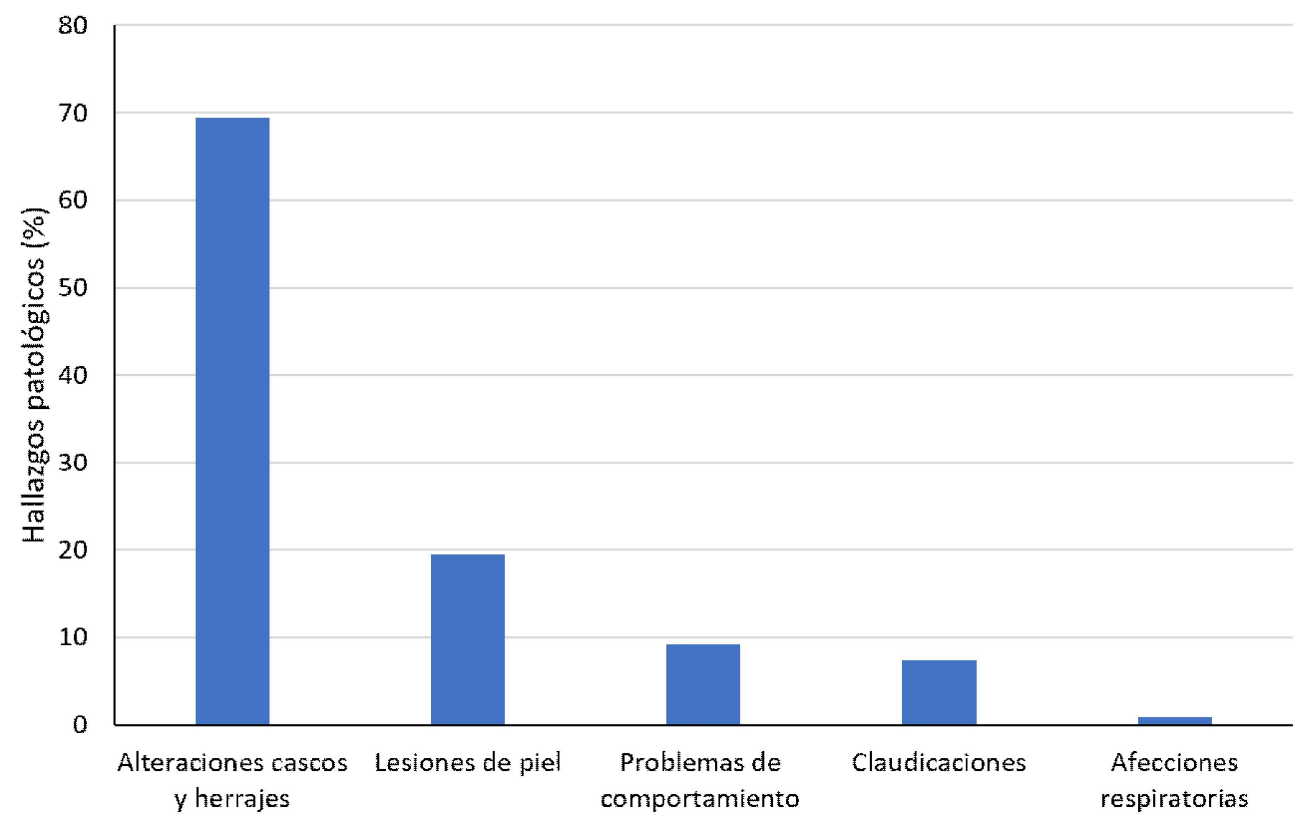

Figura 2. Caracterización de los hallazgos patológicos en equinos de tiro de comunidades Mapuche en la Región de la Araucanía, Chile.

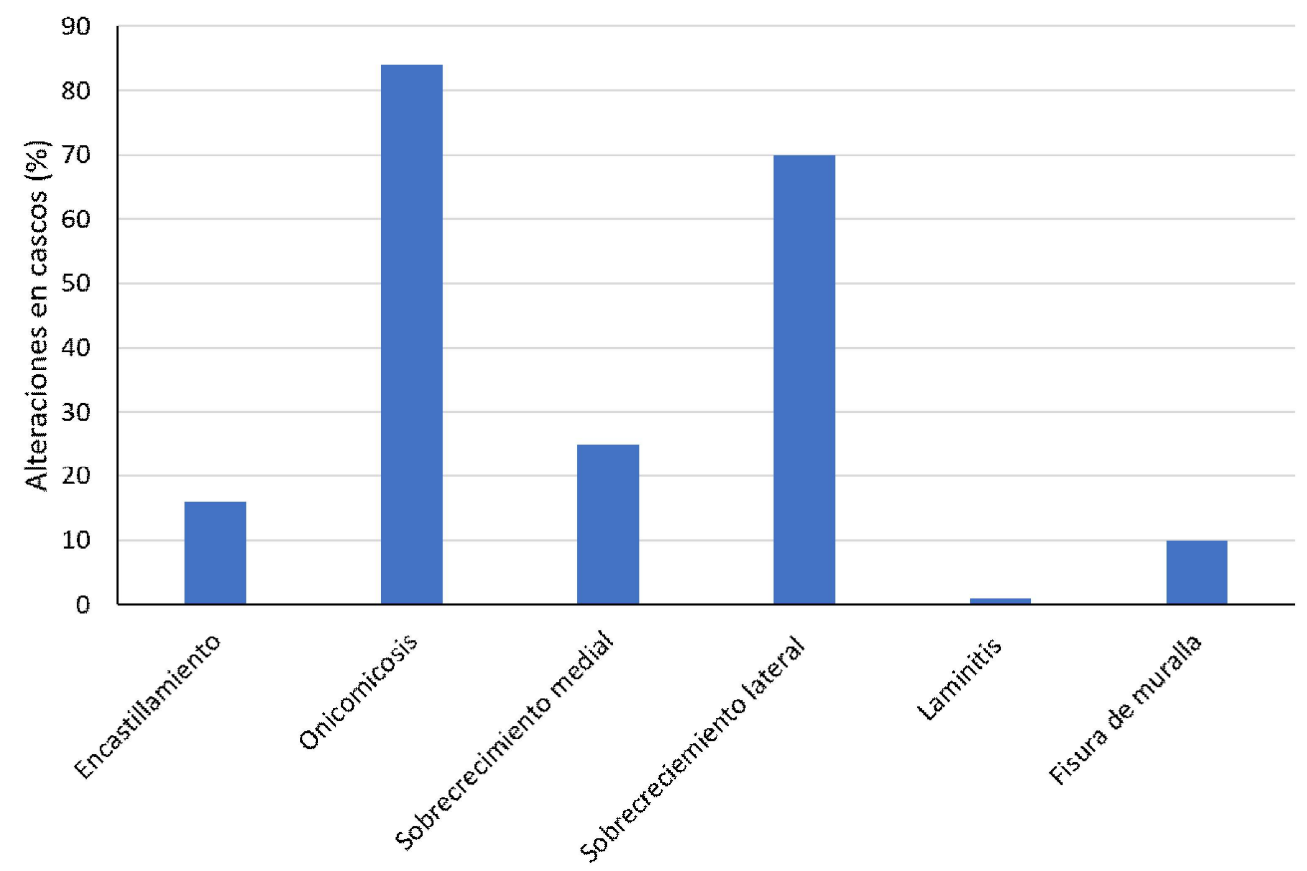

Figura 3. Alteraciones encontradas en los cascos en el grupo de equinos de tiro de comunidades Mapuche en la Región de la Araucanía, Chile. 
Cuadro 1. Alzada a la cruz en equinos de tiro de comunidades Mapuche en la Región de la Araucanía, Chile

\begin{tabular}{ccccccccc}
\hline Segmento & \multicolumn{2}{c}{ Caballos } & \multicolumn{2}{c}{ Potros } & \multicolumn{2}{c}{ Yeguas } & \multicolumn{2}{c}{ Total } \\
\cline { 2 - 9 }$(\mathrm{cm})$ & $\mathrm{n}$ & $\%$ & $\mathrm{n}$ & $\%$ & $\mathrm{n}$ & $\%$ & $\mathrm{n}$ & $\%$ \\
\hline $140-150^{*}$ & 23 & 17.2 & 10 & 7.5 & 19 & 14.2 & 52 & 38.8 \\
$150-160^{* *}$ & 34 & 25.4 & 12 & 9.0 & 34 & 25.4 & 80 & 59.7 \\
$>160^{* * *}$ & 1 & 0.8 & 1 & 0.8 & 0 & 0 & 2 & 1.5 \\
\hline Total & 58 & 43.28 & 23 & 17.16 & 53 & 39.56 & 134 & 100 \\
\hline
\end{tabular}

Se clasificaron en tres segmentos de acuerdo con Pérez et al. (1993): * caballo de silla, ** caballo de tiro liviano, ${ }^{* * *}$ caballo de tiro pesado

Cuadro 2. Perímetro torácico en equinos de tiro de comunidades Mapuche en la Región de la Araucanía, Chile

\begin{tabular}{ccccccccc}
\hline Segmento & \multicolumn{2}{c}{ Caballos } & \multicolumn{2}{c}{ Potros } & \multicolumn{2}{c}{ Yeguas } & \multicolumn{2}{c}{ Total } \\
\cline { 2 - 9 }$(\mathrm{cm})$ & $\mathrm{n}$ & $\%$ & $\mathrm{n}$ & $\%$ & $\mathrm{n}$ & $\%$ & $\mathrm{n}$ & $\%$ \\
\hline$<171^{*}$ & 25 & 18.7 & 13 & 9.7 & 22 & 16.4 & 60 & 44.8 \\
$171-181^{* *}$ & 26 & 19.4 & 7 & 5.2 & 26 & 19.4 & 59 & 44.0 \\
$>181^{* * *}$ & 7 & 5.2 & 3 & 2.2 & 5 & 3.7 & 15 & 11.2 \\
\hline Total & 58 & 43.28 & 23 & 17.16 & 53 & 39.58 & 134 & 100 \\
\hline
\end{tabular}

Se clasificaron en tres segmentos de acuerdo con Pérez et al. (1993): * caballo de silla, ** caballo de tiro liviano, $* * *$ caballo de tiro pesado

Cuadro 3. Longitud escápulo-isquial en equinos de tiro de comunidades Mapuche en la Región de la Araucanía, Chile

\begin{tabular}{ccccccccc}
\hline Segmento & \multicolumn{2}{c}{ Caballos } & \multicolumn{2}{c}{ Potros } & \multicolumn{2}{c}{ Yeguas } & \multicolumn{2}{c}{ Total } \\
\cline { 2 - 9 }$(\mathrm{cm})$ & $\mathrm{n}$ & $\%$ & $\mathrm{n}$ & $\%$ & $\mathrm{n}$ & $\%$ & $\mathrm{n}$ & $\%$ \\
\hline$<151^{*}$ & 28 & 20.9 & 13 & 9.7 & 0 & 0 & 41 & 30.6 \\
$151-162^{* *}$ & 28 & 20.9 & 10 & 7.5 & 22 & 16.4 & 60 & 44.8 \\
$>162^{* * *}$ & 2 & 1.5 & 0 & 0 & 31 & 23.2 & 33 & 24.6 \\
\hline Total & 58 & 43.27 & 23 & 17.16 & 53 & 39.57 & 134 & 100 \\
\hline
\end{tabular}

Se clasificaron en tres segmentos de acuerdo con Pérez et al. (1993): * caballo de silla, ** caballo de tiro liviano, $* * *$ caballo de tiro pesado 
Cuadro 4. Perímetro metacarpiano en equinos de tiro de comunidades Mapuche en la Región de la Araucanía, Chile

\begin{tabular}{ccccccccc}
\hline $\begin{array}{c}\text { Perímetro } \\
(\mathrm{cm})\end{array}$ & \multicolumn{2}{c}{ Caballos } & \multicolumn{2}{c}{ Potros } & \multicolumn{2}{c}{ Yeguas } & \multicolumn{2}{c}{ Total } \\
\cline { 2 - 10 } & $\mathrm{n}$ & $\%$ & $\mathrm{n}$ & $\%$ & $\mathrm{n}$ & $\%$ & $\mathrm{n}$ & $\%$ \\
\hline 19 & 5 & 3.7 & 2 & 1.5 & 0 & 0 & 7 & 5.2 \\
20 & 5 & 3.7 & 2 & 1.5 & 2 & 1.5 & 9 & 6.7 \\
21 & 22 & 16.4 & 17 & 12.7 & 26 & 19.4 & 65 & 48.5 \\
22 & 19 & 14.2 & 2 & 1.5 & 23 & 17.3 & 44 & 32.8 \\
23 & 5 & 3.7 & 0 & 0 & 2 & 1.5 & 7 & 5.2 \\
24 & 1 & 0.8 & 0 & 0 & 0 & 0 & 1 & 0.8 \\
25 & 1 & 0.8 & 0 & 0 & 0 & 0 & 1 & 0.8 \\
\hline Total & 58 & 43.29 & 23 & 17.16 & 53 & 39.55 & 134 & 100 \\
\hline
\end{tabular}

Los resultados de las medidas hipométricas de la alzada, perímetro torácico, longitud escápulo-isquial y perímetro carpiano se detallan en los cuadros 1-4.

\section{Discusión}

La distribución del sexo de los equinos destinados a la tracción animal muestra una leve tendencia al uso de machos castrados (43.2\%). Cifras distintas, pero que mantienen la tendencia, fueron registradas por Elgueta (2012), quien encontró que el 70\% de los equinos de tiro en Valdivia y Alerce (Chile) eran machos castrados. Así mismo, otros estudios realizados en el sur de Chile (Meyer, 1992; Tadich et al., 2008; Sáez et al., 2013) y en Brasil muestran similar tendencia (Maranhão et al., 2006). El uso de machos puede estar relacionado con un mayor desarrollo muscular que les permite soportar más peso y por un tiempo más prolongado. Además, se prefieren los machos castrados por su comportamiento más dócil (Tadich et al., 2008).
La edad es un factor fundamental en el bienestar de los equinos utilizados para tiro y constituye el primer acercamiento para conocer las condiciones en que se ha producido el desarrollo físico y muscular del animal (Pérez et al., 1993; Wilson, 2002) y el rendimiento en las labores de tiro (Mac-Leod, 1999). El eventual desmedro físico se relaciona con la aparición de lesiones y enfermedades en forma prematura, que tienen directa incidencia en la pérdida de confort y un bajo nivel de bienestar (Mac-Leod, 1999; Pearson et al., 2006; Tadich et al., 2008). Destinar animales zootécnicamente inmaduros, menores de 4 años, a labores de tiro puede ser perjudicial para su desarrollo (Meyer, 1992), en especial si se considera que los pesos de carga que llevan son similares a los de equinos adultos (Pearson et al., 2006). Una edad aceptable para equinos utilizados en trabajos de tiro liviano se encuentra entre 4 y 12 años (Pérez et al., 1993; Wilson, 2002). El 88.8\% de los equinos del presente estudio estuvo dentro del rango óptimo de edad y el $6.7 \%$ fue menor de cuatro años. 
La edad promedio fue de $8.5 \pm 4.4$ años, cifra que demuestra coincidencias con lo observado por Mac-Leod (1999) en la ciudad de Valdivia (7 años) y con Tadich et al. (2008) en Puerto Montt, Osorno y Valdivia (8.5 \pm 2.9 años). De la misma manera, Maranhão et al. (2006) en Brasil describen una edad promedio de 9 años y Willgert et al. (2010) en Nicaragua observaron que el $50 \%$ de los animales de tiro tenía entre 4 y 9 años.

En el estudio, el $46 \%$ de los equinos presentó una condición corporal óptima, cifra cercana a la expuesta por Willgert (2010) en equinos de tiro de Nicaragua y por Tadich et al. (2008) en el sur de Chile. Esto no significa que estén libres de alteraciones patológicas que, aunque no afecten su estado general, pueden incidir en la presentación de indicadores como el dolor, presencia de lesiones y enfermedades que comprometen el bienestar de los équidos de trabajo (OIE, 2017). En este trabajo, casi la totalidad de los propietarios adicionaban a la ingesta de pradera natural, fardos de heno de trébol y ballica, además de ofrecer grano triturado de avena en frecuencia, cantidad y horario variable, dependiendo de las posibilidades económicas.

El $80.5 \%$ de los ejemplares presentó hallazgos patológicos, siendo la mayoría relacionados con alteraciones en cascos y herrajes (69.4\%). En contraste, Mac-Leod (1999) describió hallazgos patológicos en el $38 \%$ de los animales, siendo más recurrentes las lesiones en piel. En forma similar, Tadich et al. (2008) encontraron una mayor frecuencia de lesiones de piel producidas por una mantención deficiente o utilización inapropiada de los elementos de sujeción. En el presente estudio, las lesiones de piel fueron de menor frecuencia (19.4\%), posiblemente debido a que se trató de ejemplares que se desenvolvían en un ámbito mayoritariamente rural, donde se encontraban sometidos a menos factores estresantes y donde los tiempos de trabajo eran más cortos.
Se determinó que la mayoría de los equinos del estudio no recibían un manejo podal adecuado de acuerdo con sus requerimientos. El $84 \%$ presentó onicomicosis a nivel de superficie solear y ranilla y en el $63 \%$ de los casos se hallaron deficiencias en el herraje o este era incompleto, especialmente en los miembros anteriores que se encuentran expuestos a mayor cantidad de golpes dada la irregularidad de los caminos por los que transitan. El sobrecrecimiento de cascos producto de cambios tardíos de herraduras, 0 de ausencia de estas sin los desvasados correspondientes, incide directamente sobre los ángulos que establecen el eje podofalángico y explica la presentación de claudicación en un $9 \%$ de los equinos evaluados.

La presencia de dolor y renuencia a apoyar un miembro afectado afecta el confort del animal y genera consecuencias en el comportamiento, generando conductas de evasión o agresividad, ante la tentativa de establecer la marcha (Baxter y Stashak, 2011). Esto predispone a una alta incidencia de podopatologías, en detrimento de las condiciones de vida de estos animales. Su nivel de bienestar fue bajo, similar a lo reportado por Lanas (2015).

La pradera debe proveer lo que un equino necesita, pero en este caso son animales cuya función les mantiene privados del tiempo necesario para el pastoreo (Cunha, 2012). Esto podría explicar el bajo nivel en la condición corporal encontrado en el $34 \%$ de los ejemplares, y permite comprender las variaciones que se producen en esta materia en distintas regiones del país, aun cuando la distancia entre ellas es acotada. Por otro lado, la evidencia otorgada por la descripción de los propietarios acerca de la aplicación de programas de vacunación y desparasitación permitió suponer una alta prevalencia de parasitosis, siendo este un tercer factor de importancia relacionado con la condición corporal de los animales. Esto último, además, tiene una relación directa con la condición económica de sus propietarios (Pearson et al., 2006; Upjohn et al., 2014). 
La obtención de las medidas hipométricas se relaciona directamente con la estimación del peso vivo de cada ejemplar; sin embargo, las mediciones realizadas centraron su objetivo en estimar la conformación de los ejemplares utilizados para labores de tiro, elemento de importancia en la valoración del bienestar animal (OIE, 2017). Un equino de tiro liviano debe tener las siguientes características: alzada entre 150 y 160 cm (Pérez et al., 1993), perímetro torácico entre 171 y $181 \mathrm{~cm}$, longitud escápulo-isquial entre 151 y 162 cm (Pérez et al., 1993), mientras que solo el $59.7 \%$ de los equinos de este estudio estuvo dentro del rango esperado para alzada, el 44\% para el perímetro torácico y el $44.7 \%$ para a longitud escápulo-isquial.

Por otro lado, el perímetro metacarpiano permite encontrar una coherencia y proporción adecuada entre la conformación del animal y la resistencia de sus extremidades (Pérez et al., 1993). En este estudio se observó el $81.3 \%$ de los ejemplares se situó entre 21 y $22 \mathrm{~cm}$, mientras que García et al. (1997) señalan que el perímetro metacarpiano ideal es de $19 \mathrm{~cm}$ para los caballos criollos americanos.

\section{Conclusiones}

- La principal causa del deterioro del bienestar animal en los caballos de tiro de comunidades Mapuche de la Región de la Araucanía tiene relación con deficiencias en manejos podales, debido a que no se realizan de forma adecuada ni en los tiempos necesarios.

- Menos del 50\% de los ejemplares cumplieron con las medidas hipométricas establecidas para equinos de tiro.

- Los caballos de tiro de estas comunidades no están sometidos a un programa de manejo sanitario, lo cual los predispone a enfermedades e infestaciones parasitarias que deterioran su bienestar y disminuyen su rendimiento.

\section{Literatura Citada}

1. Baxter GM, Stashak TS. 2011. Examination for lameness. In: Baxter GM(ed). Adams and Stashak's lameness in horses. $6^{\text {th }}$ ed. UK: Wiley-Blackwell. p 109-205.

2. Broom DM. 2011. Animal welfare: concepts, study methods and indicators. Rev Colomb Cienc Pec 24: 306-321.

3. Burn CC, Dennison TL, Whay HR. 2010a. Environmental and demographic risk factors for poor welfare in working horses, donkeys and mules in developing countries. Vet J 186: 385-392. doi: 10.1016/j.tvj1.2009.09.016

4. Burn CC, Dennison TL, Whay HR. 2010b. Relationship between behavior and health in working horses, donkeys and mules in developing countries. Appl Anim Behav Sci 126: 109-118. doi: 10.1016/j.applanim.2010.06.007

5. Cardona J, Álvarez J. 2018. Estimación de la edad de los caballos basado en el examen dentario. Rev UDCA Actual Divulg Cient 13: 29-39.

6. Carroll CL, Huntington PJ. 1988. Body condition scoring and weight estimation of horses. Equine Vet J 20: 41-45. doi: 10.1111/j.2042-3306.1988.tb01451.x

7. Cunha TJ. 2012. Horse feeding and nutrition. $2^{\text {nd }}$ ed. UK: Academic Press. $445 \mathrm{p}$.

8. Elgueta A. 2012. Evaluación de bienestar en equinos de tiro urbano en Valdivia y Alerce, sur de Chile. Tesis de Médico Veterinario. Valdivia: Univ. Austral de Chile. 46 p.

9. [FAOSTAT] Food and Agriculture Organization of the United Nations Statistical Databases. 2014. Life animal statistical databases. [Internet]. Available in: http://www.fao.org/faostat/ en/\#data

10. García M, Cabezas I, Guzmán R, Valenzuela S, Merino V, Pérez R. 1997. Características hipométricas, peso cor- 
poral y capacidad de carga del caballo fina sangre chileno en rodeo. Avances Cienc Vet 12: 45-47.

11. Hockenhull J, Whay HR. 2014. A review of approaches to assessing equine welfare. Equine Vet Educ 26: 159-166. doi: 10.1111/eve.12129

12. Korte SM, Olivier B, Koolhaas JM. 2007. A new animal welfare concept based on allostasis. Physiol Behav 92: 422-428. doi: 10.1016/j.physbeh.2006.10.018

13. Lanas R. 2015. Relación entre el bienestar de equinos de tiro urbano y aspectos socioeconómicos de sus propietarios en Chile. Tesis de Médico Veterinario. Santiago: Univ. de Chile. 48 p.

14. Mac-Leod C. 1999. Estudio de los equinos carretoneros atendidos en un policlínico de terreno en Valdivia, caracterizando aspectos de hipometría, patologías, alimentación, cascos y herrajes. Tesis de Médico Veterinario. Valdivia: Univ. Austral de Chile. $47 \mathrm{p}$.

15. Maranhão $R$, Palhares M, Melo M, Rezende $H$, Braga $C$, Silva $J$, Vasconcelos M. 2006. Afecções mais freqüentes do aparelho locomotor dos eqüídeos de tração no município de Belo Horizonte. Arq Bras Med Vet Zoo 58: 21-27. doi: 10.1590/S0102-09352006000100004

16. Meyer K. 1992. A study of the condition of working horses in Chile. MSc Thesis. UK: University College of North Wales. $45 \mathrm{p}$.

17. [ODEPA] Oficina de Estudios y Politicas Agrarias, Ministerio de Agricultura, Gobierno de Chile. 2015. Agricultura familiar y circuitos cortos en Chile: situación actual, restricciones y potencialidades [Internet]. Disponible en: http:/ /www.indap.gob.cl/docs/default-source/ default-document-library $/ \mathrm{n} \% \mathrm{C} 2 \% \mathrm{BA} 1-$ archivo-orig.pdf?sfvrsn $=0$

18. [OIE] Organización Mundial de Salud Animal. 2017. Código sanitario para los animales terrestres. Cap. 7. Bienestar de los animales. [Internet]. Disponi- ble en: http://www.oie.int/index.php? id $=169 \& \mathrm{~L}-=2 \&$ htm file $=$ t itre $1.7 . \mathrm{htm}$

19. Palmer C, Sandoe P. 2011. Animal ethics. In: Appleby MC, Mench JA, Olsson IA, Hughes B (eds). Animal welfare. $2^{\text {nd }}$ ed. London: CABI. p 1-12.

20. Pearson RA, Krecek RC. 2006. Delivery of health and husbandry improvements to working animals in Africa. Trop Anim Health Pro 3: 93-101. doi: 10.1007/s11250-006-4363-y

21. Pérez R, Cabezas I, Guzmán R, García M, Chavarría C, Soto R. 1993. Comparación de características hipométricas de caballos descendientes de potros fina sangre y criollos de tiro. Avances Cienc Vet 8: 1-2. doi: 10.5354/07195273.2010.6128

22. Pritchard JC, Lindberg AC, Main DC, Whay HR. 2005. Assessment of the welfare of working horses, mules and donkeys, using health and behaviour parameters. Prev Vet Med 69: 265-283. doi: 10.1016/j.prevetmed.2005.02.002

23. Requejo C. 2010. La protección penal de la fauna. Especial consideración del delito de maltrato de animales. Sevilla, España: Comares. 168 p.

24. Rimbaud E, Caballero P, Morales $X$, Soto JL, Rivera G, Zepeda N, Gutierrez, $M$, et al. 2006. Situación de la salud de cascos, boca y piel de los caballos de cocheros y carretoneros correspondientes a la alcaldía de Granada, Nicaragua. REDVET 7(12). [Internet]. Disponible en: https://www.redalyc.org/articulo.oa?id $=63612664012$

25. Sáez M, Escobar A, Tadich TA 2013: Morphological characteristics and most frequent health constraints of urban draught horses attending a free healthcare programme in the south of Chile: a retrospective study (1997-2009). Livestock Res Rural Dev 25(91). [Internet]. Disponible en: https:// www.1rrd.cipav.org.co/lrrd25/5/ saez25091.htm 
26. Swann WJ. 2006 Improving the welfare of working equine animals in developing countries. Appl Anim Behav Sci 100: 148-151. doi: 10.1016/j.applanim. 2006.04.001

27. Tadich T. 2006. Husbandry and welfare aspects of urban draught horses in the south of Chile. MSc Thesis. Scotland: University of Edinburgh. $72 \mathrm{p}$.

28. Tadich T, Escobar, Pearson RA. 2008. Aspectos de manejo y bienestar en equinos de tiro urbano en el sur de Chi1e. Arch Med Vet 40: 267-273. doi: 10.4067/S0301-732X2008000300007

29. Upjohn MM, Pfeiffer DU, Yerheyen KLP. 2014. Helping working Equidae- and their owners in developing countries: monitoring and evaluation of evidencebased interventions. Vet J 199: 210-216. doi: 10.1016/j.tvj1.2013.09.0657

30. Willgert E. 2010. Impact of veterinary assistance on the health of workinghorses in Nicaragua. Swedish University of Agriculture Science. [Internet]. Available in: https://stud.epsilon.slu.se/ 1891/1/willgert_e_101020.pdf

31. Wilson R. 2002. Specific welfare problems associated with working horses. In: Waran N (ed). The welfare of horses. Animal Welfare Series $\mathrm{N}^{\circ} 1$. USA. p 203-218. 